\title{
The Cardiovascular Benefits of Polyphenol Compounds
}

\author{
Anthony San Luis \\ University of North Carolina at Chapel Hill, Chapel Hill, USA \\ Email: Asanluis@Live.unc.edu
}

Received February 28 $8^{\text {th }}, 2013$; revised March 29 ${ }^{\text {th }}, 2013$; accepted May $1^{\text {st }}, 2013$

\begin{abstract}
Copyright (c) 2013 Anthony San Luis. This is an open access article distributed under the Creative Commons Attribution License, which permits unrestricted use, distribution, and reproduction in any medium, provided the original work is properly cited.
\end{abstract}

\begin{abstract}
In comparison, polyphenols are one of the most diverse to most other groups of bioactive phytochemicals. Polyphenols can be found in a wide range of foods that are derived from plants. Polyphenols are made up of various subgroups, but it's the phenolics, stillbenes, and flavonoids that engender positive impacts on cardiovascular health. These three phytochemical compounds can reduce cardiovascular conditions such as hypertension and coronary heart disease. Remarkably, polyphenols and its subgroups can be found ubiquitously in most human diets. As a result, many studies have effectively illustrated the positive impacts polyphenols can induce when processed by the human body. Furthermore, research efforts have shown that modern diets and more "traditional" diets have unknowingly promoted the consumption of foods rife with polyphenols. These findings are starting to be incorporated into the public health discipline as a more practical option for more sustainable nutritional interventions. In all, polyphenols can produce a myriad of positive developments on at the micro and macro level.
\end{abstract}

Keywords: Phytochemicals; Stillbenes; Phenolics; Flavonoids Formatting; Style; Styling

\section{Introduction}

With great success, humans have been able to manage an exceptional level of coevolution with an extensive array of neighboring organisms. Of the myriad of extant relationships that humans have experienced throughout our history, the most intriguing and important to our survival has been the association with our botanical counterparts. It is this particular instance of symbiosis that is probably the most visible, as well as one of the oldest co-evolutionary relationships that humans have cultivated. Plants have been central to the survival of our ancestral species, and in a sense many species of plants echo these same sentiments. If not for the arrival of the human species, the evolutionary trajectory of many species of plants would not have been able to survive. As humans have evolved into the "ultimate" survivalists over several millennia, plants have been utilized in a way central to their survival.

Historically speaking, humans have been afforded a high level of achievement when it comes to the modification of plants in some fashion. It is not out of the realm of possibility that dating as far back as our australopithecine ancestors were able to effectively deduce and segregate ideally ripe fruits and vegetables based on phenotypically desirable characteristics. This simple relationship is the genesis of a communicative relationship between humans and plants that have engendered subsequent interactions that would benefit both organisms for centuries. As early as the agricultural revolution, humans have favorably manipulated this dyadic relationship to a point that the question emerges as to whom is actually manipulating whom. For instance, human cultures have manipulated grapes and yeast to make wine close to seven thousand years ago (Thompson, 2005). The question remains as to whether humans are using the fermentation process to benefit them or are the grapes and yeasts using humans to cultivate and till more fields dedicated for their own progress to perpetuate their species. However, the extent of human and plant coevolution resonates deeper than merely farming techniques, rather it requires a look at the molecular level.

At the molecular level plants contain bioactive phytochemicals and when in contact with the human body can produce adverse and beneficial reactions. Plants have developed bioactive phytochemicals as a chemical defense response to the environmental pressures and stresses (Kryzyzanowska et al., 2010). In particular, compounds such as phenolics, carotenoids, alkaloids, and saponins etc. have played crucial functions in the survival of the plant species but simultaneously given humans an opportunity to benefit from its consumption. Humans invariably have enjoyed high levels of advances when extracting these bioactive phytochemicals for their own use. Furthermore, bioactive phytochemical extraction has been exercised by humans to produce a number of health benefits. Traditional Chinese medicine is probably one of the first examples of its accomplishments that have been present over several centuries and it is starting to become common in cultures outside of China. The interaction of bioactive phytochemicals and biomedicine has engendered a litany of positive results that are being explored in depth as it relates to the human species' ability to survive. In allopathic medicine, the phytochemical compounds extracted from plants have played roles in traditional medicine and ethnopharmacology for humans to effectively alleviate and in some cases, cure several diseases.

Specifically, bioactive phytochemical compounds have been efficacious as serving in a cardio-protective capacity. Humans have been able to derive positive influences in a number of settings contributing to overall well-being. Several fruits and vegetables contain these bioactive compounds that directly influ- 
ence cardiovascular health and have been investigated intensely to substantiate this notion. Epidemiological studies of the role of tomatoes that are rich sources of lycopene were studied in a comparison between a cohort of Lithuanian and Swedish populations (Tucker, 2004). This study showed that high lycopene levels reduce morbidity and mortality from coronary heart disease (Kryzyzanowska et al., 2010). However, this is just the beginning of the seemingly infinite range of effects that can lower the risk of cardiovascular diseases. This is because many fruits and vegetables have a diverse content of bioactive phytochemicals compounds linked with the promotion of cardiovascular health.

\section{Aims and Purposes}

In this paper, I discuss and review the literature on the effects of certain bioactive phytochemicals found in a range of fruits and vegetables that promote cardiovascular health. The role of the phytochemical polyphenol and constituent subgroups phenolics, stillbenes, and flavonoids are reviewed for their properties to promote cardiovascular health. In a sense, I will take a pragmatic approach that will depart from the biochemistry literature to a review of the publications demonstrating research efforts about types of dietary interventions that now focus on phytochemicals. Specifically, this will entail a description of modern and traditional dietary approaches addressing hypertension. A review of the Dietary Approach to Stop Hypertension (DASH) model shows that research efforts focusing on the incorporation of bioactive phytochemicals has become wholly integrated into various dietary interventions. This paper review will conclude with a recommendation towards public health interventions. I will analyze public health benefits that can be produced by the consumptive habits stemming from fruits and vegetables that contain significant cardio-protective properties.

\section{Analysis \\ Introduction of Polyphenols}

Polyphenols are one of the most diverse and as a result, one of the most relevant groups of phytochemicals (Gonzales-Castejon \& Rodriguez-Casado, 2011). Such diversity is illustrated by the various subgroups that constitute the polyphenol family due to being endowed with a wide range of beneficial impacts on health. Additionally, research has shown that polyphenols demonstrate strong protective effects on several pathological conditions that are facilitated by oxidative stress like cardiovascular disease and metabolic syndrome through the aid of antiangionic properties that suppress the growth of adipose tissue and the modulation of adipocyte metabolism (Gonzales-Castejon \& Rodriguez-Casado, 2011). Polyphenols are mostly known through its numerous subgroups having one hydroxyl-substituted aromatic ring (Gonzales-Castejon \& Rodriguez-Casado, 2011). However, of all the variability that exists in the polyphenol group, its particular subgroups have made the polyphenol group all the more intriguing, namely phenolics, stillbenes, and flavonoids all enjoy properties that are conducive to cardiovascular health.

\section{Phenolics}

Within the polyphenol group, its subgroup phenolics (or simple phenolic acids) are the largest and arguably the most important constituents of polyphenols when compared to its counter- parts. While simple phenolic acids like caffiec acid, chologenic acid, and feluric acid are some examples of the compounds that makeup the phenolic subgroup have all been found in dietary sources like coffee (Coffea Arabica) and soybeans (Glycine so$j a)$ as well as fruits and vegetables in concentrated levels in apples (Malus domestica), and pineapples (Anana comosus) (Gonzales-Castejon \& Rodriguez-Casado, 2011). Although these sources of phenolic acids have cardiovascular protective properties, catechin has been the most closely studied type of phenolic and has been able to traverse geographical barriers into the minds of individuals across several cultures and populations because of the ubiquitous consumption of a green tea (Camellia sinesis) that been discovered to be a source rich in catechin.

The highest concentrations of catechins are found in green tea that has been known to exhibit positive impacts on cardiovascular health. Green tea is the most strikingly effective phenolic directly linked with the prevention and/or alleviation of conditions associated with cardiovascular disease through the promotion of several biological mechanisms. A type of catechin, epigallocatechin gallate (EGCG), has a large concentration in green tea and has been substantiated to be more efficacious in addressing these conditions. The mechanism of actions of green tea and EGCG have been found to have direct impacts on the biological function of the body to address cardiovascular diseases. EGCG has shown to address obesity and lower the risk of hypertension by reducing adipocyte differentiation and proliferation, fat mass, body weight, fat absorption, insulin, while simultaneously stimulating thermogenic activity (Tucker, 2004). Comparatively speaking, the content of EGCG of green tea in comparison to its next closest dietary source is impressive to say the least. Dark chocolate is engendered with a large amount EGCG with $53.5 \mathrm{mg} / 100 \mathrm{~g}$ but green tea more than doubles this amount with a $132 \mathrm{mg} / 100 \mathrm{~g}$ making it a more desirable choice when opting for purer sources of EGCG (Kryzyzanowska et al., 2010: p. 84). The benefits of the consumption of plants and vegetables rich in catechins were validated by a trial studying the intake of green tea. The ensuing results showed that a standardized intake of catechins through the consumption of green tea showed that there was a decrease in total body weight and waist circumference caused by an increase in thermogenesis (Gonzales-Castejon \& Rodriguez-Casado, 2011).

\section{Stillbenes}

Stillbenes, along with positive impacts on cardiovascular health, also has a potent effect in-vitro and is found in a large array of dietary plant sources. Similar to most polyphenolics, stillbenes also occur naturally but in limited sources, it is mostly found in large quantities in several varieties of fruit like apples, cranberries, blueberries, and grapes. Resveratrol is found in stillbenes and has been the key factor directly linked with the prevention of hypertension. Resveratrol is often found in high concentrations in grapes but levels are actually higher in wine because of the production process and have been shown to limit risk of cardiovascular diseases.

Although, it is the content of resveratrol that is found in wine that produces positive effects on the heart, its biological processes are additionally influenced by the presence of a geographical component that alters its quantities. Stillbenes and its constituent resveratrol are synthesized by the body through phenolynpropanoid pathways in response to environmental stress, infections, disease, and ultraviolet exposure (Gonzales-Castejon 
\& Rodriguez-Casado, 2011). Resveratrol levels found in wine have are known to have direct cardiovascular effects as an antihypertensive because of its anticoagulant properties that limit platelet aggregation (Kryzyzanowska et al., 2010). Specifically, resveratrol has been able to decrease LDL-cholesterol and prevent lipid oxidation while producing favorable changes in gene expression and enzyme activity involved in metabolic processes (Gonzales-Castejon \& Rodriguez-Casado, 2011). However, varieties of wine (red vs. white) contain varying levels of resveratrol and are limited to certain constraints that influence its resveratrol levels and its impact because of the presence of limiting factors that favor one country's production over another. Certain countries like Italy are endowed with an agricultural component that increases resveratrol levels in wine because of its production, and technological innovations that have immense influence on the resveratrol levels in the wine coming out of this area (Tucker, 2006). For example, wines consumed by Italian males have significantly higher proportions of resveratrol when compared to wines from other countries. In Italian wines, resveratrol levels are nearly .5 - $10 \mathrm{ppm}$, whereas wine produced in Japan for example, has resveratrol rates of .05 - .8 ppm (Tomera, 1999). Therefore, it can be assumed that Italians and other consumers who have access to wine produced in Italy would benefit from a greater antihypertensive effect compared to the consumption of wine from elsewhere.

\section{Flavonoids}

Like its polyphenol counterpart's stillbenes and phenols, flavonoids also engender positive impacts on cardiovascular health. Over 6000 distinct phytochemicals have been identified in fruits and vegetables, with many current research efforts focusing on its anti-inflammatory properties that have can address many chronic diseases (Gonzales-Castejon \& Rodriguez-Casado, 2011). As a result, the vegetable and fruit sources of flavonoids can vary greatly. Nonetheless, their positive impacts on cardiovascular diseases are becoming known and providing profound benefits from seemingly "common" vegetables and fruits.

Alone, the content of flavonoids cannot explain the beneficial effects derived from its consumption, but rather it is the content of the compounds like quercetin, kaempferol, myricetin, and anthocyanidin that provides flavonoids as viable compounds to prevent and alleviate heart conditions. Some of the more common sources of flavonoids are often found in fruitbased sources like grapefruit (Citrus sinensis) and blueberries (Cyanococcus vaccinum) but have also been found in high concentrations in vegetable-based sources like broccoli (Brassica oleracea), and leeks (Allium porrum) (Gonzales-Castejon \& Rodriguez-Casado, 2011; Dillar \& German, 2000). Flavonoid biological function encompasses a variety of different mechanisms with positive impacts in addressing cardiovascular conditions such as obesity or diabetes. These compounds have been linked to a decrease in platelet aggregation and thrombosis that ultimately lowering the risk of coronary heart disease (Dillar \& German, 2000). Additionally, other outcomes have shown that these flavonoid compounds can induce the inhibition of LDL oxidation, and lowering blood pressure (Gonzales-Castejon \& Rodriguez-Casado, 2011).

Many studies have effectively substantiated these effects through the identification, isolation, and administration of flavonoid rich diets in a number of trials attempting to investigate its beneficial properties. In a longitudinal trial in 1985 with 805 elderly males, recorded daily baseline intake of flavonoid levels measuring $25.9 \mathrm{mg}$ found that the relationship of the intake between flavonoid intake and coronary heart disease mortality was inversely related (Dillar \& German, 2000). The results confidently claimed that a consistent consumption of a flavonoidrich diet can have positive efficacy on not just males, but elderly males specifically. Further, studies have also shown that the cardiovascular health of women improved with a consistent level of consumption of broccoli and apples as well (Tucker, 2004).

In trials that were recently reviewed by Katherine Tucker for the Human Nutrition Research Institute of Aging and Marta Gonzales-Castejon \& Arantxa Rodriguiz-Casado for the Pharmacological Research have demonstrated the efficacy of flavonoids for its protective properties against cardiovascular diseases have been widely demonstrated through various publications. Diets emphasizing the consumption of flavonoids are becoming gradually showing its viability in dietary options to promote healthy well-being. The fact that flavonoids are relatively common in most dietary settings produces ostensibly easy access to its consumption and its cardiovascular benefits barring any limiting extraneous variables. Specifically in most settings both in a traditional and modern sense, fruits like grapefruit and vegetables like broccoli are pervasive in most market settings, and ought to be consumed consistently for both their immediate and long-term preventative impacts.

The consumption of foods rich in polyphenols has clearly illustrated its effects on the human cardiovascular system. Polyphenol-rich foods have pervaded the human diet with great success with the arrival of globalization and mainstream media promoting its benefits. Even in the face of certain barriers that could limit its accessibility such as socioeconomic status or food insecurity, products like Italian-made wine have been able to make the journey across the Atlantic Ocean away from Italy into the kitchens of many US residents. This sentiment is echoed in green tea that originated in east Asia has also managed to penetrate the restaurant and supermarket business with seemingly relative ease in recent decades. It would come as great benefit for humans to consistently utilize foods containing polyphenols for their consumption because its impacts have been demonstrated adequately by empirical research. It seems virtually inevitable that more research illustrating its impact are on the horizon as more studies begin to study the place of all known bioactive phytochemicals in mainstream dietary interventions that promote cardiovascular health.

\section{Discussion}

\section{The Intersection of Phytochemicals with Contemporary and Traditional Dietary Approaches Promoting Cardiovascular Health}

Several dietary interventions in various settings have been tasked with directly addressing cardiovascular health. Many modern dietary interventions trying to address cardiovascular disease by lowering rates of obesity have been achieved but in traditional settings absent of westernized medicine have been explained also have been able to reduce the risk of cardiovascular diseases. Few contemporary dietary interventions, if any have used a comprehensive analysis that looks into the contribution of bioactive phytochemicals compounds. There have been ex- 
amples of modern approaches such as the Dietary Approaches to Stop Hypertension (DASH) that promote the consumption of diverse but specific range of foods that implicate a protection against cardiovascular conditions. On the contrary, in the absence of modern science and research settings, rural traditional communities have been recently found to utilize the extraction phytochemical compounds from plants indigenous to that region to proliferate cardiovascular health.

The DASH diet has been able to lower the risk of hypertension in individuals who have adhered to its recommendations. The DASH diet is starting to become more recognized by healthcare officials as an adequate option in the dietary management of hypertension. But research efforts have delved further into the factors lending to its accomplishments beyond a simple modification of diet by exploring the effects of the food that is recommended in the DASH diet (Most, 2004). The composition of the DASH diet promotes the consumption of fruits, vegetables, and low-fat dairy foods in order to reduce blood pressure and is also known to protect against lipid peroxidation. Essentially, the DASH diet is one such intervention that aims to effectively combat hypertension through sustained alterations of an individual's diet but not until recently, the phytochemical content present in the DASH diet have been identified as key factors in its ability to lower hypertension.

A recent study by the Department of Agriculture compiled a database of the recommended foods in the DASH diet and its phytochemical makeup to highlight their place as a key contributor to preventing hypertension. The contents of phytochemical compounds such as flavonoids, carotenoids, flavanones, and phytoesterols were some of the focal points for their study in comparison to a control diet. The results showed a drastic disparity in the amounts of these phytochemical compounds found in the DASH diet than in the control diet (Most, 2004, 1726). The results underscored that the DASH diet versus the control diet had a higher concentration (mg/1000 kcal) of flavonols (3.53 vs. 2.24), carotenoids (12.24 vs. 3.81), flavonones (6.77 vs. .44), and phytoesterols (47.19 vs. 19.22 ) respectively (Most, 2004).

On the other hand, without the use of modern scientific approaches characterized by modern interventions, more traditional communities have been just as rewarding with the utilization of certain indigenous plants. In rural Uganda, the leaf of the Moringa oleifera plant was initially touted by local Ugandan media in the 1980's as a purported cure to alleviate symptoms linked to HIV/AIDS. However, the Moringa oleifera plant has found its uses expand into other areas of health, namely cardiovascular health. In a trial studying its effects, have been able to show that Moringa oleifera has a moderately high concentration of flavonols. Subsequently an impressive $90 \%$ of males in these rural Ugandan communities have consistently consumed its leaves to treat diabetes and hypertension without actually knowing its effects that have been substantiated by research efforts (Kasolo et al., 2010).

Both of these recent findings produce a unique perspective with implications on the evolutionary history of humans. The DASH diet mostly concentrated on the diversified consumption of foods containing macronutrients and micronutrients. But the findings produced in the rural Ugandan example illustrate that dietary interventions may have been part of the natural course of human innovation regardless of the advances made in modern western medicine. Yet, the underlying sentiment remains, that if diets knowingly or in the case of rural the Ugandan community unknowingly, emphasizing a biochemical focus would stand to have more positive impacts on overall wellbeing. The DASH diet in particular, with a focus on phytochemicals can broaden its scope of the DASH philosophy to include the plants like Moringa oleifera among many other plants that have yet been identified or are under current investigation. A focus on the biochemical reactions of phytochemicals would give not just the DASH diet but other dietary interventions new opportunities to produce a level of affirmation generated by empirical results that is often the basis for acceptance in many institutional settings.

\section{Public Health Implications of Using Phytochemicals to Address Hypertension and Other Cardiovascular Conditions}

The public health paradigm have been extremely concerned with the nutritional status of entire populations, and in recent decades have tried to implement nutritional interventions with varying levels of success. In the case of cardiovascular health, public health has struggled mightily to effectively produce a "universal" cure in the face of rising rates of obesity and malnutrition. However, many of these interventions can be costly for those involved, and at times have not been able to produce a positively uniformed impact. However, a "universal” cure is not necessarily what is needed, but perhaps a universal effort focused on future sustainability. It appears that there are costeffective techniques to address the extant cases of malnutrition stemming from the existence of poor quality food in impoverished populations in countries that have experienced rapidly development where rates chronic and infectious disease rising.

One recommendation would require the use of a more nuanced approach with a level of cultural sensitivity by public health officials to integrate an "organic" or "natural” facet into the diet, aiming towards a more agriculturally sustainable focus on certain phytochemicals, while at the same time, avoiding synthetic means of production such as genetically modified organisms (GMO's), steroids, etc. A combination of a public health perspective and an anthropological approach geared towards biochemistry could be implemented to effectively narrow the gap in dietary management of hypertension. Examples taken from the DASH diet or the Ugandan use of Moringo oleifera can demonstrate that there are other ways to address malnutrition beyond the reliance of commercial pharmaceutical companies producing synthetic products. A biological anthropological approach would comprehensively consider the cultural specificities and tailor diets based on a particular population's consumption of certain vegetables and fruits. This would call for the cultivation of native plants with phytochemicals indigenous to a particular region. Essentially cultivating fruits and vegetables rich in certain bioactive phytochemicals with high concentrations of polyphenol compounds like phenolics, stillbenes, and flavonoids could promote cardiovascular health with the goal of also providing a level of sustainability for communities with high rates cardiovascular disease.

\section{Concluding Remarks}

The trajectory of human evolution has entailed the cultivation of various relationships with a sundry of organisms. Yet, the relationship with plants has been one with the longest history whose dyadic association is still producing new results 
seemingly every day but at the same time with a large remainder still a mystery in the eyes of modern science. Dating back to prehistory with our ancestral hominids up till the agricultural revolution thousands of years ago humans are often thought in some circles to be selectively manipulating plants for the survival and proliferation of their biological fitness. Additionally, recent research has shown that plants are composed of specific phytochemicals often with a myriad of benefits to the human body. As a result, the health implications associated with the consumption of phytochemical compounds found in plants has been studied intensely and largely continues in investigative settings across the world. These findings have been able to traverse several disciplines beyond agriculture into public health and disciplines in between, where many efforts are beginning to study the effectiveness of bioactive phytochemical compounds for nutritional purposes on larger scales. It remains to be seen if these efforts are going to be effective at broad level, but the focus remains on the betterment of the species not just society towards a more sustainable environment for future generations to comfortably exist.

\section{REFERENCES}

Asaolu, M. F., Asaolu, S. S., \& Adanlawo, I. G. (2010). Evaluation of phytochemicals and antioxidants of four botanicals with anti-hyper- tensive properties. International Journal of Pharma and Bio Sciences, $1,1-7$.

Dillard, C., \& Bruce, G. (2000). Phytochemicals: Nutraceuticals and human health. Journal of Science Food and Agriculture, 80, 17441756.

http://dx.doi.org/10.1002/1097-0010(20000915)80:12<1744::AID-JS FA725>3.0.CO;2-W

Gonzales-Castejon, M., \& Arantxa, R.-C. (2011). Dietary phytochemicals and their potential effects on obesity. Pharmacological Research, 64, 438-455. http://dx.doi.org/10.1016/j.phrs.2011.07.004

Kasalo, J., Gabriel, B., Lonzy, O., \& Jasper, O.-O. (2010). Phytochemicals and uses of moringa leaves in ugandan rural communities. Journal of Medicinal Plants Research, 4, 753-757. http://dx.doi.org/10.5897/JMPR10.492

Most, M. (2004). Estimated phytochemical content of the dietary approaches to stop hypertension (DASH) diet is high than in the contro study diet. Journal of the American Dietetic Association, 1726-1728. http://dx.doi.org/10.1016/j.jada.2004.08.001

Thompson, J. (2005). The geographic mosaic of coevolution. Chicago, IL: University of Chicago Press.

Tomera, J. (1999). Current knowledge of the health benefits and disadvantages of wine consumption. Trends in Food and Science, 10, 129138.

Tucker, K. (2004). Dietary intake and coronary heart disease: A variety of nutrients and phytochemicals are important. Current Treatment Options in Cardiovascular Medicine, 6, 291-302. http://dx.doi.org/10.1007/s11936-004-0031-6 